\title{
Single Particle CryoEM: Potential for Further Improvement
}

\author{
Richard Henderson $^{1 *}$ and Christopher J. Russo ${ }^{1}$ \\ 1. MRC Laboratory of Molecular Biology, Cambridge CB2 0QH, UK \\ * Corresponding author: rh15@mrc-lmb.cam.ac.uk
}

Recent progress in single particle electron cryo-microscopy (cryoEM) has allowed determination of the structures of a range of novel macromolecules or macromolecular assemblies, many of which have proved intractable to other approaches. The method requires only a few tens of micrograms of material; the sample does not have to be completely pure; nor completely stable. The success of the approach has been built on the early development of a plunge-freezing method for producing a thin film of amorphous ice [1] in which biological structures could be embedded [2], and by three-dimensional (3D) structure determination algorithms that have evolved out of early random conical tilt [3] and angular reconstitution [4] approaches. The final quantum leap in capability came from technical improvements in the electron microscopes together with better detectors and improvements in computing [5]. The most popular microscope platforms at present, such as the Thermo Fisher Titan Krios or the JEOL CryoARM300, are still very expensive, and there is not yet enough capacity to cope with the demand. This means it is difficult for smaller groups not located in major research universities to obtain access to modern cryoEM equipment, so there is an urgent need for more affordable cryoEM.

We have also been analysing the technical limitations that prevent cryoEM from reaching its ultimate potential. We have made quantitative evaluations of the candidate problems: we have shown that charge build up on non-conducting thin films of amorphous ice [6], the "Berriman effect", occurs on a shorter timescale than image acquisition, that microscopic charge fluctuations that can be observed as the "beeswarm effect" do not significantly degrade the image quality [7], and confirmed [8] that corrections for Ewald sphere curvature proposed earlier [9] remove one of the arguments for using higher energy electrons. Alongside measurements of the effects of radiation damage, this has allowed us to propose a rough physical theory of information loss in cryoEM [10]. It is summarized in a model (Fig. 1) of how the B-factor decays with electron fluence during imaging. The B-factor is the Debye-Waller factor, which can be used to assess information content [11]. The key outstanding problem is the elimination of mechanical beam-induced specimen motion in the early part of a typical cryoEM image acquisition (shaded area in Fig. 1). Although gold grids with thick holey gold supporting foils [12] offer a significant reduction in motion, there is still a critical and uncontrolled electron beam-induced motion in the very earliest part of the exposure, where the specimen structure is least damaged by electron irradiation.

Lastly, we have analysed which aspects of current electron microscope technology are really essential for cryoEM, with the goal of making it less expensive and widely accessible. From an analysis of elastic and inelastic cross-sections and radiation damage [13], we have concluded that a $100 \mathrm{keV}$ cryoEM equipped with a bright (coherent) electron source and a good detector could be as good (or even better for many purposes) as the best current technology, yet could be much less expensive. New ideas are therefore needed to address the remaining problem of specimen motion in the earliest part of low-dose exposures, and to develop instruments that are more affordable yet still allow the great majority of cryoEM experiments to be performed [14]. 
References:

[1] J Dubochet et al. J. Microscopy 128 (1982) p. 219.

[2] J Dubochet et al. Quarterly Reviews of Biophysics 21 (1988), p. 129.

[3] M Radermacher et al. J. Microscopy 146 (1987), p. 113.

[4] M van Heel, Ultramicroscopy 21 (1987), p. 111.

[5] W Kuhlbrandt, Science 343 (2014) p. 1443.

[6] CJ Russo and R Henderson, Ultramicroscopy 187 (2018), p. 43.

[7] CJ Russo and R Henderson, Ultramicroscopy 187 (2018), p. 56.

[8] CJ Russo and R Henderson, Ultramicroscopy 187 (2018), p. 26.

[9] M Wolf, DJ DeRosier and N Grigorieff, Ultramicroscopy 106 (2006), p.376.

[10] CJ Russo and R Henderson, to be published.

[11] PB Rosenthal and R Henderson, J. Mol. Biol. 333 (2003) 721.

[12] CJ Russo and LA Passmore, Science 346 (2014), p. 1377.

[13] MJ Peet, R Henderson and CJ Russo, Ultramicroscopy 203 (2019), p. 125.

[14] The authors acknowledge funding from the UK Medical Research Council grant numbers MC_U105184322 and MC_UP_1201/17. We thank our colleagues Mathew Peet, Greg

McM̄ullan, Wasi Faruqi and Shäoxia Chen for discussions and collaborations, and the Medical Research Council for support.

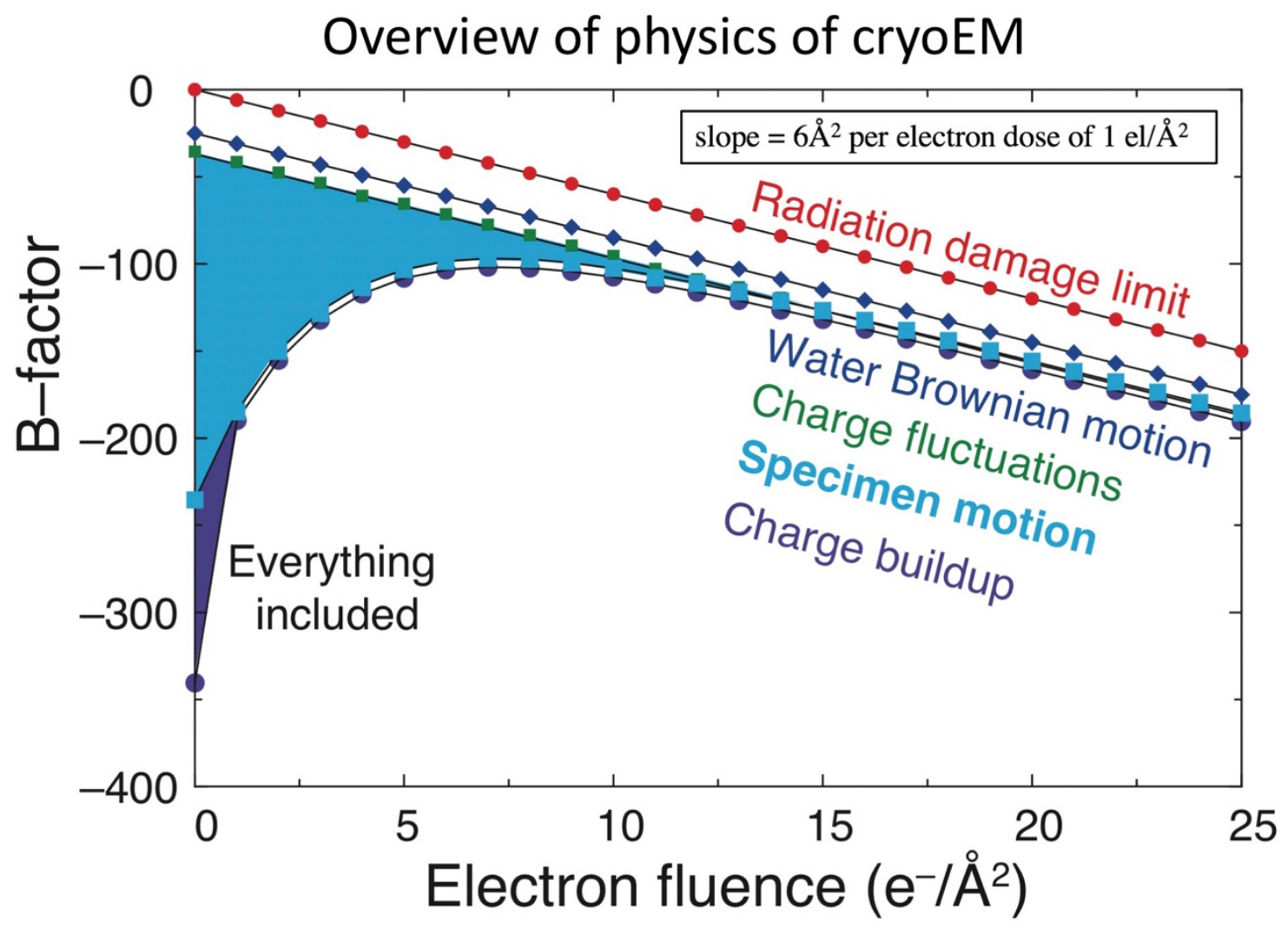

Figure 1. Summary of the effects on B-factor as a function of electron fluence of five different possible sources of information loss in single particle cryoEM [10]. The most important is mechanical specimen motion (shaded area) during the first part of the exposure at electron doses up to about $5 \mathrm{e}^{-/}$ $\AA^{2}$. 